\title{
Nasal functional modifications after physical exercise: olfactory threshold and peak nasal inspiratory flow*
}

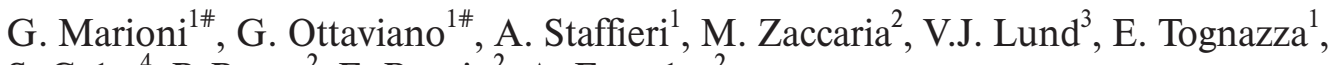

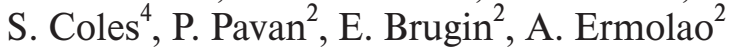 \\ 1 Department of Medical and Surgical Specialties, Section of Otolaryngology, University of Padova, Padova, \\ Italy \\ 2 Department of Medical and Surgical Sciences, Sports Medicine Unit, University of Padova, Padova, Italy \\ 3 The Ear Institute, University College London, London, United Kingdom \\ ${ }^{4}$ Department of Statistical Sciences, University of Padova, Padova, Italy
}

SUMMARY

\begin{abstract}
Statement of problem: The respiratory nasal effects of physical exercise have been extensively investigated; on the other hand there are no data regarding olfactory threshold modification after aerobic physical exercise.

Methods: The present prospective study investigated the modifications in nasal respiratory flows and olfactory thresholds after controlled aerobic physical exercise in a cohort of 15 adult, healthy volunteers. The Peak Nasal Inspiratory Flow (PNIF), and the Sniffin' Sticks olfactory threshold test were used for our determinations.

Main results: The mean PNIF after physical exercise was significantly higher than the mean PNIF value found before physical exercise. Statistical analysis ruled out any significant difference between mean olfactory thresholds pre vs post physical exercise.

Principal conclusions: These outcomes confirmed PNIF sensitivity and reliability also in determining the changes in nasal patency occurring after physical exercise. The active vasoconstriction of nasal mucosa associated with the reduction of blood flow to the olfactory epithelium due to physical exercise may be compensated for by the increase of olfactory molecules that reach the olfactory mucosa because of nasal mucosal shrinkage: this mechanism could explain the stability of mean olfactory threshold after physical exercise.
\end{abstract}

Key words: nasal function, physical exercise, olfactory threshold, Sniffin' Sticks, Peak Nasal Inspiratory Flow (PNIF)

\section{INTRODUCTION}

The respiratory nasal effects of physical exercise have been extensively investigated. Exercise causes a decrease in nasal mucosal congestion similar to that seen with the application of a nasal decongestant such as oxymetazoline hydrochloride ${ }^{(1)}$. Overall, exercise promotes a drop in total nasal airway resistance within 30 seconds that is maximal at 5 minutes and may persist for up to 30 minutes after completing the aerobic performance $^{(1)}$.

On the other hand, there is a significant dearth of information regarding olfaction modification during and after aerobic physical exercise.

The aim of the present prospective study was to investigate the change in nasal respiratory flow and olfactory thresholds after controlled aerobic physical exercise in a cohort of 15 adult, healthy volunteers. We used for our measurements two sim- ple, objective and reliable clinically emerging modalities: 1) the Peak Nasal Inspiratory Flow (PNIF), and 2) the Sniffin' Sticks olfactory threshold test, that is available since $1996^{(2)}$ and is nowadays one of the most widely used olfactory tests in Europe.

\section{MATERIALS AND METHODS \\ Volunteers}

Fifteen Caucasian volunteers ( 8 males and 7 females) aged from 20 to 33 years (mean age $26.2 \pm 3.6$ years; median age 27.0 years) were recruited. All subjects were healthy and moderately active but not involved in professional or competitive sports activities. The approval of a written informed consent was required to participate to the study. Each subject was asked to complete a SNOT 20 (Sinonasal Outcome Test) ${ }^{(3)}$ to asses nasal symptoms. To be included in the present study, the volunteers had to score less than 1 on the SNOT 20, had to be

\# The first two authors equally contributed to study design and manuscript preparation. 
neither a smoker nor asthmatic and had to report no previous sinonasal surgery. Other inclusion criteria were no history of cardiovascular, pulmonary, or orthopedic diseases, and a normal body mass index $\left(<25.0 \mathrm{~kg} / \mathrm{m}^{2}\right)$. All the subjects who satisfied the inclusion criteria were asked about medications (none of them took medications); a full physical examination was performed. The present investigation complied with the current laws of Italy, and was approved by local institutional review board.

\section{Testing program}

Once definitively included into the investigation, all volunteers were tested twice by the same group of trained investigators. The first evaluation was performed at least 1 week before the second test. During the 2 days before the first and second evaluations, the volunteers were asked to eat a balanced diet, to avoid coffee, drugs, alcoholic drinks, and exhaustive exercise, and to maintain a regular sleeping pattern. On test days, the subjects came to the laboratory in the afternoon (at least two hours after lunch), waiting a 20-minute acclimatisation period before the exam was carried out. Laboratory temperature was maintained between 20 and $22^{\circ} \mathrm{C}$. Maximal oxygen uptake was determined measuring breath-by-breath gas exchange (CSD/Net System 2001, Medical Graphic Corporation, Minnesota, USA) by a treadmill test (Marquette T-2000 series, General Electrics, Waukesha, WI, U.S.A.) using a standardized incremental ramp protocol. $\mathrm{VO}_{2} \max$ determination criteria were subjects exhaustion (Borg Scale $>18 / 20$ ), and at least two of these maximal effort criteria: $\mathrm{V}_{2}$ plateau, defined as a small or no increase in $\mathrm{VO}_{2}$ in response to an increase in treadmill speed, a respiratory exchange ratio $(\mathrm{RER})>1.10$, a difference between the maximal heart rate recorded and the age-predicted maximal heart rate lower than 10 beats/min. Anaerobic threshold was determined using the ventilatory equivalent method.

Before starting the treadmill test, we measured basal PNIF and olfactory thresholds for n-butanol. To measure PNIF, we used a portable Youlten peak flow meter (Clement Clark International, Harlow, UK). Recording the PNIF values, volunteers were encouraged to inhale as hard and fast as they could through the nose with the mouth tightly closed and the mask firmly over the face, starting from the end of a full expiration. All subjects were tested while sitting. According to previous experiences reported by Ottaviano et al. ${ }^{(4)}$, for each volunteer we obtained two satisfactory maximal inspirations and the highest value of the two inspirations was taken as basal PNIF value. As we usually do for clinical purposes, smell ability was tested by a Le Nez du Vin as a quick test of olfaction. The volunteers that gave $\leq 1$ wrong answers with the Le Nez du Vin test underwent the Sniffin' Sticks test (Burghart Medical Technology, Wedel, Germany) only for the determination of olfactory threshold for $n$-butanol. The Sniffin' Sticks test battery is characterised by 16 dilutions prepared in geometric series starting from a $4 \% n$-butanol solution (dilution ratio 1:2 in deionised aqua conservata as solvent) ${ }^{(6)}$. Odorants were presented in felt-tip pens; pen triplets were presented in a randomized order, with two containing the solvent and the third the odorant. Triplets were presented at intervals of approximately 20 seconds ${ }^{(6)}$. When measuring olfactory thresholds, subjects were blindfolded to prevent visual identification of the odorant-containing pens. Olfactory threshold scores ranged between 1 and 16 .

After the basal nasal study, the treadmill test was started. A prolonged exhaustive exercise was performed, where, after 1 minute of warm-up, subjects run for 10 minutes at the speed corresponding to their previously determined anaerobic threshold. After this period, the speed was progressively increased by $10 \%$ every 30 seconds, until subject's exhaustion. Immediately after physical activity, PNIF and olfactory threshold for n-butanol were measured again according to the previously described modality.

\section{Statistical analysis}

To compare pre vs post physical exercise nasal respiratory flows and olfactory thresholds the Wilcoxon test, which avoids parametric assumptions, has been used. A p-value $<0.05$ was considered to be significant; values in the range $0.10>p \geq 0.05$ were considered as indicating a statistical trend. R: A Language and Environment for Statistical Computing (R Foundation for Statistical Computing, Vienna, Austria) was used to execute the Wilcoxon test on matched pairs and Fisher's z-test while power calculations were made using the $\mathrm{G}^{*}$ Power 3 package ${ }^{(7)}$.

\section{RESULTS}

All volunteers completed the planned exercise tests. The mean duration of the second prolonged maximal exercise test was 13 minutes and 7 seconds \pm 59 seconds (range: 11 minutes 0 seconds -14 minutes 39 seconds). The average PNIF before physical exercise was $176.0 \pm 50.8 \mathrm{1} / \mathrm{min}$ (median value 170.0 $1 / \mathrm{min}$ ). The average PNIF immediately after physical exercise was $222.0 \pm 68.7 \mathrm{l} / \mathrm{min}$ (median value $200.0 \mathrm{l} / \mathrm{min}$ ). The Wilcoxon test found that PNIF determined after physical exercise was significantly higher than PNIF determined before physical exercise $(\mathrm{p}=0.000356)$.

None of the volunteers gave more than one wrong answer at the Le Nez du Vin test, used to evaluate smell ability, thus, all volunteers underwent olfactory threshold for n-butanol determination based on the Sniffin' Sticks test battery. According to Hummel et al., ${ }^{(6)}$ olfactory threshold data analysis and average olfactory thresholds were calculated. The average olfactory threshold for n-butanol before physical exercise was $13.4 \pm 5.1$. The average olfactory threshold after physical exercise was 13.3 \pm 5.3. The Wilcoxon test ruled out any significant difference between mean olfactory thresholds pre vs post physical exercise $(p=0.540)$. Because of the failure of the latter test to identify any pre vs post difference, calculations were made to assess the approximate power of the test procedure used. Assuming 
temporarily that data are normally distributed, and maintaining a matched pair test at the 5\% level of significance with a sample of 15 patients, the power of the test in identifying a difference of 2 in the mean level of the pre and post population is $91.0 \%$. Such a high level of power suggests that the test failure to obtain a significant difference between the pre vs post olfactory results is more likely to be due to a genuine lack of difference rather than a limitation of test discriminatory power.

Sample correlations between pre-exercise PNIF and olfactory threshold, post-exercise PNIF and olfactory threshold, and the differences between pre- and post-exercise for the two phenomena were $0.173,0.313$, and 0.325 , respectively (Fisher's ztest).

\section{DISCUSSION}

Several factors would be involved in the action of physical exercise on the reduction of nasal resistance: increase in the activity of alar nasal muscle, blood redistribution for muscles under exercise distant from nasal mucosa, increase in nasal airflow, hyperventilation, and active vasoconstriction of the nasal mucosa ${ }^{(8)}$. Autonomic innervation involvement in modifying nasal patency has been widely accepted ${ }^{(9)}$. Konno and co-workers ${ }^{(10)}$ measured change of serum norepinephrine (NE) level and nasal patency in 10 healthy volunteers during exercise and evaluated the effect of unilateral cervical sympathetic ganglion block on exercise-induced shrinkage of nasal mucosa. Serum NE levels grew markedly after 10-min exercise. Unilateral blocking of cervical ganglion completely inhibited mucosal shrinkage induced by exercise in almost all subjects. These findings supported the hypothesis that mucosal shrinkage during exercise was more probably due to mediation of cervical sympathetic ganglion than to the increase in circulating catecholamines. In 1997, Lacroix et al. ${ }^{(11)}$, studying in a cohort of healthy volunteers the variations of plasma concentrations of neuropeptide Y (NPY), a vasoconstrictor neurotransmitter peptide co-localized with NE in perivascular sympathetic nerves, found that plasma concentrations of NPY correlated with post-exercise nasal vasoconstriction more strictly than NE. These authors concluded that NPY might act as a modulator of nasal airways reactivity ${ }^{(11)}$. Several authors compared nasal patency before and after physical exercise using different approaches mostly based on plethysmography, anterior ${ }^{(12,13)}$ or posterior rhinomanometry ${ }^{(14,15)}$, acoustic rhinometry ${ }^{(8,16)}$, and nasal ozone uptake ${ }^{(17)}$. Nasal modifications after controlled physical exercise were not previously investigated by PNIF, which is a cheap, simple and easily performed method to assess nasal patency ${ }^{(4,18)}$. In the present cohort, statistical analysis showed that mean PNIF value determined after physical exercise $(222.01 / \mathrm{min})$ was significantly higher than mean PNIF value found before physical exercise (170.0 1/min). The present outcome clearly confirmed PNIF sensitivity and reliability also in determining the changes in nasal patency, which occurred after physical exercise.
Only limited data are available regarding the effect of exercise on special senses (hearing, smell) associated to ear, nose and throat structures. In particular, evaluating the auditory system, the Staffieri and di Prampero research group at Udine University ${ }^{(19)}$ found in a limited cohort of volunteers that aerobic physical exercise induced a temporary threshold shift. This temporary threshold shift could be explained by catecholamine release, which induced splanchnic vasoconstriction with a consequent reduction of blood flow towards cochlear outer hair cells. In an animal model (miniature swine), Delp et al. ${ }^{(20)}$ found that blood flow to the olfactory bulbs and rhinencephalon, which are central components of the olfactory system, were not elevated during physical exercise. On the contrary, during exercise vascular resistances were elevated in the rhinencephalon. These findings supported the hypothesis that olfactory neural activities were not heightened during dynamic exercise.

The present study investigated the effect of a short duration (between 11 and 15 minutes) maximal exercise on the olfactory threshold. We chose a maximal exercise to induce a significant rise in catecholamine plasma concentrations, as well as to elicit an adequate cardiovascular adaptation and a maximal drop in total nasal airway resistance. A cohort of 15 healthy Caucasian volunteers was recruited. All volunteers gave $\leq 1$ wrong answers using the smell ability test Le Nez du Vin and underwent determination of olfactory threshold. For this we opted for "Sniffin' Sticks", a test of nasal chemosensory function that is based on pen-like odour dispensing devices, introduced in 1996 by Kobal and co-workers ${ }^{(2)}$. The use of this test has been endorsed by the "Working Group on Olfaction and Gustation" of the German Society for Otorhinolaryngology, Head and Neck Surgery ${ }^{\left({ }^{6}\right)}$. All our volunteers homogeneously belonged to the age group B (16-35 years) of the recently published normative data for the "Sniffin' Sticks" including olfactory thresholds ${ }^{(6)}$. Although we calculated a high mean olfactory threshold in the considered series before and after exercise, nothing was preliminary done to select the volunteers according to their olfactory thresholds. During data collection we tried, besides following the precepts established for the treadmill test, the Le Nez du Vin test and olfactory threshold determination, to avoid external factors that could interfere in its accuracy, limiting temperature variations (less than $2^{\circ} \mathrm{C}$ ), acclimatising the volunteers before testing, and having the exams performed by the same trained team of investigators. Statistical analysis ruled out any significant difference between mean olfactory thresholds pre vs post physical exercise. The present outcome may be explained by the fact that the active vasoconstriction of nasal mucosa demonstrated during physical exercise could be associated with a similar reduction of blood flow also to the olfactory cells. The effect of the blood flow reduction on olfaction could be compensated by the increase of olfactory molecules that reach the olfactory mucosa because of widely reported nasal mucosal shrinkage. These changes, 
determining opposite effects on smell perception, could explain why mean olfactory thresholds pre vs post physical exercise were not significantly different. The preliminarily found stability of mean olfactory threshold after physical exercise requires further prospective verification in larger series settings, considering also the fact that olfactory threshold may take longer to change than the timeframe we used in the present investigation.

\section{REFERENCES}

1. Baraniuk JN, Merck SJ. Nasal reflexes: implications for exercise, breathing, and sex. Curr Allergy Asthma Rep. 2008; 8: 147-153.

2. Kobal G, Hummel T, Sekinger B, et al. "Sniffin' sticks": screening of olfactory performance. Rhinology. 1996; 34: 222-226.

3. Piccirillo JF, Merritt MG Jr, Richards ML. Psychometric and clinimetric validity of the 20-Item Sino-Nasal Outcome Test (SNOT20). Otolaryngol Head Neck Surg. 2002; 126: 41-47.

4. Ottaviano G, Scadding GK, Coles S, Lund VJ. Peak nasal inspiratory flow. Normal range in adult population. Rhinology. 2006; 44: 32-35.

5. McMahon C, Scadding GK. Le Nez du Vin-a quick test of olfaction. Clin Otolaryngol Allied Sci. 1996; 21: 278-280.

6. Hummel T, Kobal G, Gudziol H, et al. Normative data for the "Sniffin' Sticks" including tests of odor identification, odor discrimination, and olfactory thresholds: an upgrade based on a group of more than 3000 subjects. Eur Arch Otorhinolaryngol. 2007; 264: 237-243.

7. Faul F, Erdfelde E, Lang A-G, et al. G*Power 3: A flexible statistical power analysis program for the social, behavioral, and biomedical sciences. Behav Res Methods. 2007; 39: 175-191.

8. Fonseca MT, Machado JA, Pereira SA, et al. Effects of physical exercise in nasal volume. Braz J Otorhinolaryngol. 2006; 72: 256260.

9. Dallimore NS, Eccles R. Changes in human nasal resistances associated with exercise, hyperventilation and rebreathing. Acta Otolaryngol. 1977; 84: 416-421.

10. Konno A, Togawa K, Itasaka Y. Neurophysiological mechanism of shrinkage of nasal mucosa induced by exercise. Auris Nasus Larynx. 1982; 9: 81-90.

11. Lacroix JS, Correira F, Fathi M, et al. Post-exercise nasal vasoconstriction and hyporeactivity: possible involvement of neuropeptide Y. Acta Otolaryngol. 1997; 117: 609-613.
12. Portugal LG, Mehta RH, Smith BE, et al. Objective assessment of the breathe-right device during exercise in adult males. Am J Rhinol. 1997; 11: 393-397.

13. Wilde AD, Ell SR. Effect of nasal resistance of an external nasal splint and isotonic exercise. Br J Sports Med. 1999; 33: 127-128.

14. Strohl KP, Decker MJ, Olson LG, et al. The nasal response to exercise and exercise induced bronchoconstriction in normal and asthmatic subjects. Thorax. 1988; 43: 890-895.

15. Wheatley JR, Amis TC, Engel LA. Nasal and oral airway pressureflow relationships. J Appl Physiol. 1991; 71: 2317-2324.

16. Uzzaman A, Metcalfe DD, Komarow HD. Acoustic rhinometry in the practice of allergy. Ann Allergy Asthma Immunol. 2006; 97: 745-751.

17. Sawyer K, Brown JS, Hazucha MJ, et al. The effect of exercise on nasal uptake of ozone in healthy human adults. J Appl Physiol. 2007; 102: 1380-1386.

18. Ottaviano G, Lund VJ, Coles $\mathrm{S}$, et al. Does peak nasal inspiratory flow relate to peak expiratory flow? Rhinology. 2008; 46: 200-203.

19. Miani C, Bertino G, Francescato MP, et al. Temporary threshold shift induced by physical exercise. Scand Audiol. 1996; 25: 179186.

20. Delp MD, Armstrong RB, Godfrey DA, et al. Exercise increases blood flow to locomotor, vestibular, cardiorespiratory and visual regions of the brain in miniature swine. J Physiol. 2001; 533.3: 849859.

Gino Marioni, MD

Department of Medical and Surgical Specialties Section of Otolaryngology

University of Padova

Via Giustiniani 2, 35128

Padova

Italy

Tel. +39-(0)49-821 8626

Fax: +39-(0)49-821 3113

E-mail: gino.marioni@unipd.it 